\title{
Molecular Tracing of SARS-CoV-2 in Italy in the First Three Months of the Epidemic
}

\author{
Alessia Lai ${ }^{1, *}$, Annalisa Bergna ${ }^{1}$, Sara Caucci ${ }^{2}$, Nicola Clementi ${ }^{3}$, Ilaria Vicenti ${ }^{4}$ (D), \\ Filippo Dragoni ${ }^{4}$, Anna Maria Cattelan ${ }^{5}$, Stefano Menzo ${ }^{2}$, Angelo Pan ${ }^{6}$, Annapaola Callegaro ${ }^{7}$, \\ Adriano Tagliabracci ${ }^{8}$ (D), Arnaldo Caruso ${ }^{9}$, Francesca Caccuri ${ }^{9}$, Silvia Ronchiadin ${ }^{10}$ (D), \\ Claudia Balotta ${ }^{1}$, Maurizio Zazzi ${ }^{4}$ (D), Emanuela Vaccher ${ }^{11}{ }^{\mathbb{D}}$, Massimo Clementi ${ }^{3}$, \\ Massimo Galli ${ }^{1}$ and Gianguglielmo Zehender ${ }^{1}$ on behalf of SARS-CoV-2 ITALIAN RESEARCH \\ ENTERPRISE-(SCIRE) Collaborative Group (Appendix A)
}

1 Department of Biomedical and Clinical Sciences Luigi Sacco, University of Milan, 20157 Milan, Italy; bergna.anna@gmail.com (A.B.); claudia.balotta@unimi.it (C.B.); massimo.galli@unimi.it (M.G.); gianguglielmo.zehender@unimi.it (G.Z.)

2 Department of Biomedical Sciences and Public Health, Virology Unit, Polytechnic University of Marche, 60131 Ancona, Italy; s.caucci@univpm.it (S.C.); menzo@univpm.it (S.M.)

3 Microbiology and Virology Unit, "Vita-Salute" San Raffaele University, 20132 Milan, Italy; clementi.nicola@hsr.it (N.C.); clementi.massimo@hsr.it (M.C.)

4 Department of Medical Biotechnologies, University of Siena, 53100 Siena, Italy; ilariavicenti@gmail.com (I.V.); dragonifilippo@gmail.com (F.D.); maurizio.zazzi@gmail.com (M.Z.)

5 Infectious Diseases Unit, Department of Internal Medicine, Azienda Ospedaliera-Universitaria di Padova, 35128 Padua, Italy; annamaria.cattelan@aopd.veneto.it

6 Infectious Diseases, ASST Cremona, 26100 Cremona, Italy; a.pan@asst-cremona.it

7 Microbiology and Virology Laboratory, ASST Papa Giovanni XXIII, 24127 Bergamo, Italy; acallegaro@hpg23.it

8 Section of Legal Medicine, Universita Politecnica delle Marche, 60126 Ancona, Italy; a.tagliabracci@staff.univpm.it

9 Microbiology Unit, Department of Molecular and Translational Medicine, University of Brescia and ASST Spedali Civili Hospital, 25123 Brescia, Italy; arnaldo.caruso@unibs.it (A.C.); francesca.caccuri@unibs.it (F.C.)

10 Intesa Sanpaolo Innovation Center-AI LAB, 10138 Turin, Italy; silvia.ronchiadin@intesasanpaolo.com

11 Medical Oncology and Immune-related Tumors, Centro di Riferimento Oncologico di Aviano (CRO), IRCCS, 33081 Aviano, Italy; evaccher@cro.it

* Correspondence: alessia.lai@unimi.it; Tel.: +39-0250319775

Received: 3 July 2020; Accepted: 21 July 2020; Published: 24 July 2020

\begin{abstract}
The aim of this study is the characterization and genomic tracing by phylogenetic analyses of 59 new SARS-CoV-2 Italian isolates obtained from patients attending clinical centres in North and Central Italy until the end of April 2020. All but one of the newly-characterized genomes belonged to the lineage B.1, the most frequently identified in European countries, including Italy. Only a single sequence was found to belong to lineage B. A mean of 6 nucleotide substitutions per viral genome was observed, without significant differences between synonymous and non-synonymous mutations, indicating genetic drift as a major source for virus evolution. tMRCA estimation confirmed the probable origin of the epidemic between the end of January and the beginning of February with a rapid increase in the number of infections between the end of February and mid-March. Since early February, an effective reproduction number $\left(R_{e}\right)$ greater than 1 was estimated, which then increased reaching the peak of 2.3 in early March, confirming the circulation of the virus before the first COVID-19 cases were documented. Continuous use of state-of-the-art methods for molecular surveillance is warranted to trace virus circulation and evolution and inform effective prevention and containment of future SARS-CoV-2 outbreaks.
\end{abstract}


Keywords: Phylodynamic analyses; SARS-CoV2 circulation in Italy; molecular tracing; whole genome sequencing

\section{Introduction}

Italy is one of the countries most- and earliest-affected in Europe by the COVID-19 pandemic (https: //gisanddata.maps.arcgis.com/apps/opsdashboard/index.html\#/bda7594740fd40299423467b48e9ecf6). The first autochthonous cases of Coronavirus 2019 Disease (COVID-19) were observed starting from 21 February 2020 in Codogno (Lodi province), determining on 22 February 2020 the establishment of a "red zone" to contain the epidemic, encompassing 11 municipalities. Thereafter, in a short time, it became evident that the epidemic had already involved a large part of Lombardy region and then spread to neighboring regions and, substantially less, to the rest of the country. On 9 March lockdown was declared for the entire country. The rapidly increasing number of patients who required hospitalization in the intensive care unit suggested that the virus may have circulated for a long period and caused thousands of contagions before the epidemic became manifest [1].

SARS-CoV-2 was first detected in Italy in a couple of Chinese tourists coming from Wuhan on 31 January [2]. Subsequent evaluations have not shown a relationship between the sequence of these strains and those implicated in the epidemic in Lombardy [3].

On the contrary, the Codogno strains resulted strictly related with a strain of SARS-CoV-2 coming from Shanghai which caused a small outbreak in Munich around 20 January [1] and was probably spread later to other European countries and beyond the Atlantic [4]. These sequences are part of a clade initially defined as a European clade, the old Nexstrain A2a subclade, which is currently the most widespread outside China and probably responsible for most of the world pandemic [5].

In the face of more than 240,000 notified cases in Italy, the entire genomes available in public databases are still scarce (77 at the time of this study). The availability of large numbers of sequences collected over time is necessary for molecular surveillance of the epidemic and for evaluation and planning of effective control strategies. To perform this study, a network of Italian Clinical centres and Laboratories across Italy generated additional 59 full-length SARS-CoV-2 sequences from COVID-19 patients ranging from the end of February to the end of April. This contribution helps to trace the temporal origin, the rate of viral evolution and the population dynamics of SARS-CoV-2 in Italy by phylogeny.

\section{Materials and Methods}

\subsection{Patients and Methods}

A total of 59 SARS-CoV-2 whole genomes were newly-characterized from an equal number of patients affected by COVID-19, attending different clinical centres in Northern and Central Italy, from the beginning of the epidemic (22 February 2020) until 27 April 2020 (Table S1).

All of the data used in this study were previously anonymized as required by the Italian Data Protection Code (Legislative Decree 196/2003) and the general authorizations issued by the Data Protection Authority. Ethics Committee approval was deemed unnecessary because, under Italian law, all sensitive data were deleted and we collected only age, gender and sampling date (Art. 6 and Art. 9 of Legislative Decree 211/2003).

Eighteen sequences were obtained after isolating the virus in Vero E6 cells while the remaining 41 were obtained directly from biological samples such as nasopharyngeal swabs or broncho-alveolar lavages (39 and 2, respectively).

SARS-CoV-2 RNA was extracted using the Kit QIAsymphony DSP Virus/Pathogen Midi kit on the QIAsymphony automated platform (QIAGEN, Hilden, Germany) $(n=9)$ and manually with QIAamp Viral RNA Mini Kit (QIAGEN, Hilden, Germany) $(n=50)$. 
Full genome sequences were obtained with different protocols by amplifying 26 fragments as previously described $(n=42)$ [1] or using random hexamer primers $(n=8)$ or Ion AmpliSeq SARS-CoV-2 Research Panel (Thermo Fisher Scientific, Waltham, Massachusetts, USA) $(n=9)$. The PCR products were used to prepare a library for Illumina deep sequencing using a Nextera XT DNA Sample Preparation and Index kit (Illumina, San Diego, California, USA) in accordance with the manufacturer's manual, and sequencing was carried out on a Illumina MiSeq platform for 50 samples, while the remaining nine were sequenced on Ion GeneStudio ${ }^{\mathrm{TM}}$ S5 System instrument following the Ion AmpliSeq ${ }^{\mathrm{TM}}$ RNA libraries protocol (Thermo Fisher Scientific, Waltham, Massachusetts, USA). The results were mapped and aligned to the reference genome obtained from GISAID (https: //www.gisaid.org/, accession ID: EPI_ISL_412973) using Geneious software, v. 9.1.5 (Biomatters, Auckland, New Zealand) (http://www.geneious.com) [6] or Torrent Suite v. 5.10.1 (Euformatics Oy, Espoo, Finland) or BWA-mem and rescued using Samtools alignment/Map (Hinxton, UK) (v 1.9).

\subsection{Sequence Data Sets}

The newly-characterized 59 genomes plus three previously characterized isolates by us (EPI_ISL_417445-417447) [1] were aligned with a total of 77 Italian sequences available in public databases (GISAID, https://www.gisaid.org/) on 13 May 2020 and 452 genomes sampled in different European and Asian countries (513 and 16, respectively) representing all the different viral clades described in the Nextstrain platform (https://nextstrain.org/). The final data set thus included 588 sequences. Due to the large amount of available sequences, we focused the analysis on European strains by randomly selecting sequences from each country and by excluding identical strains or strains with more than $5 \%$ of gaps. We sampled the data in order to have no temporal gaps, by grouping the sequences by country/week/clade and randomly selecting the sequences in each group. We choose 15 sequences for clade A2 and 5 sequences for other clades (therefore including A and B Pangolin lineages and different sublineages) for each European country. For countries with less than the required sequence number we kept all the sequences. The sampling dates of the entire dataset ranged from 30 December 2019 to 27 April 2020. Table S2 shows the accession IDs, sampling dates and locations of the sequences included in the dataset.

A subset of sequences assigned to the old Nextstrain A2 clade (classified as B lineage for Pangolin), was generated for dating the epidemic, including all the Italian sequences, one German (EPI_ISL_406862) and three Chinese isolates from Shanghai, ancestral to the A2 clade (EPI_ISL_416327, EPI_ISL_416334 and EPI_ISL_416386). Coalescent and birth-death phylodynamic analyses were performed on the 136 Italian A2 sequences only.

Alignment was performed using MAFFT [7] and manually cropped to a final length of 29,779 bp using BioEdit v. 7.2.6.1 (http://www.mbio.ncsu.edu/bioedit/bioedit.html).

\subsection{Genetic Distance, Recombination, and Selection Pressure Analyses}

The MEGA X program was used to evaluate the genetic distance between and within Italian sequences on the full length genome, with variance estimation performed using 1000 bootstrap replicates [8].

The RDP5 software was used to investigate the presence of potential recombination [9].

All of the genes were tested for selection pressure using Datamonkey (https://www.datamonkey. org/). Amino acid changes were evaluated using EPI_ISL_402123 as reference strain.

\subsection{Phylogenetic and Phylodynamic Analyses}

The simplest evolutionary model best fitting the sequence data was selected using the JmodelTest v.2.1.7 software [10], and proved to be the Hasegawa-Kishino-Yano model with a proportion of invariant sites $(\mathrm{HKY}+\mathrm{I})$.

The phylogenetic analysis for clade assignment was performed by RaxML [11] on the entire dataset of 588 genomes. During the period in which we were carrying out the study, the SARS-CoV-2 
clade nomenclature system changed. In particular, Rambaut et al. proposed a dynamic nomenclature based on phylogenetic lineages, called Pangolin (Phylogenetic Assignment of Named Global Outbreak LINeages) [12]. For this reason, we used the old Nextstrain and the new Pangolin (freely available at https: //pangolin.cog-uk.io/) systems for strain classification. The new Nextstrain classification was performed by using the available script (https://github.com/nextstrain/ncov/blob/master/docs/running.md).

The virus' phylogeny, evolutionary rates, times of the most recent common ancestor (tMRCA) and demographic growth were co-estimated in a Bayesian framework using a Markov Chain Monte Carlo (MCMC) method implemented in v.1.10.4 and v.2.62 of the BEAST package [13,14].

A root-to-tip regression analysis was made using TempEst in order to investigate the temporal signal of the dataset [15].

Different coalescent priors (constant population size and exponential growth and Bayesian skyline) and strict vs. relaxed molecular clock models were tested by means of path sampling (PS) and stepping stone (SS) sampling [16]. The evolutionary rate prior normal distribution, after informing the mean evolutionary rate, was set at mean $0.8 \times 10^{-3}$ substitutions/site/year (http: //virological.org/t/phylodynamic-analysis-176-genomes-6-mar-2020/356).

The MCMC analysis was run until convergence with sampling every 10,000 generations. Convergence was assessed by estimating the effective sampling size (ESS) after 10\% burn-in using Tracer v.1.7 software (http://ree.bio.ed.ac.uk/software/tracer/), and accepting ESS values of 200 or more. The uncertainty of the estimates was indicated by $95 \%$ highest marginal likelihoods estimated [17] by path sampling/stepping stone methods [16].

The final trees were summarized by selecting the tree with the maximum product of posterior probabilities (pp) (maximum clade credibility or MCC) after a 10\% burn-in using Tree Annotator v.1.10.4 (included in the BEAST package), and were visualized using FigTree v.1.4.2 (http://tree.bio.ed. ac.uk/software/figtree/). Posterior probabilities $>0.7$ were considered significant.

\subsection{Birth-Death Skyline Estimates of the Effective Reproductive Number $\left(R_{e}\right)$}

The birth-death skyline model implemented in Beast 2.62 was used to infer changes in the effective reproductive number $\left(R_{e}\right)$, and other epidemiological parameters such as the death/recovery rate $(\delta)$, the transmission rate $(\lambda)$, the origin of the epidemic, and the sampling proportion $(\rho)$ [18]. Given that the samples were collected during a short period of time, a "birth-death contemporary" model was used.

The analyses were based on the previously selected HKY substitution model and the evolutionary rate was set to the value of $0.8 \times 10^{-3} \mathrm{subs} / \mathrm{site} / \mathrm{year}$, which corresponds to the mean substitution rate estimated using a relaxed clock under the exponential coalescent model as transformed into units per year.

For the birth-death skyline analysis, from one to two $R_{e}$ intervals and a log-normal prior with a mean (M) of 0.0 and a variance $(S)$ of 1.0 were chosen, which allows the $R_{e}$ values to change between $<1(0.193)$ to $>5$. A normal prior with $\mathrm{M}=48.7$ and $\mathrm{S}=15$ (corresponding to a $95 \%$ interval from 24.0 to 73.4) was used for the rate of becoming uninfectious. These values are expressed as units per year and reflect the inverse of the time of infectiousness (5.3-19 days, mean 7.5) according to the serial interval estimated by Li et al. [19]. Sampling probability $(\rho)$ was estimated assuming a prior Beta (alpha $=1.0$ and beta $=999$ ), corresponding to a minority of the sampled cases (between $10^{-5}$ to $10^{-3}$ ). The origin of the epidemic was estimated using a normal prior with $\mathrm{M}=0.1$ and $S=0.05$ in units per year.

The MCMC analyses were run for 100 million generations and sampled every 10,000 steps.

Convergence was assessed on the basis of ESS values (ESS > 200). Uncertainty in the estimates was indicated by $95 \%$ highest posterior density (95\%HPD) intervals.

The mean growth rate was calculated on the basis of the birth and recovery rates $(r=\lambda-\delta)$, and the doubling time was estimated by the equation: doubling time $=\ln (2) / \mathrm{r}[20]$. 


\section{Results}

\subsection{Phylogenetic Analysis of the Whole Dataset}

No recombination events were observed in the entire dataset according to analyses with RDP5 software.

Phylogenetic analysis by maximum likelihood showed that the Italian sequences were included in a single SARS-CoV-2 clade (the old Nextstrain A2 clade, corresponding to new Nextstain clades 20A and 20B) with the exception of three sequences: Two from Chinese patients visiting Italy at the end of January 2020 after being infected in Wuhan and one characterized by us from an Italian subject, living in Padua, sampled in March 2020, not reporting any recent trip outside Italy or contacts with subjects affected by COVID-19 (pp = 0.99) (Figure 1, clade 19A).
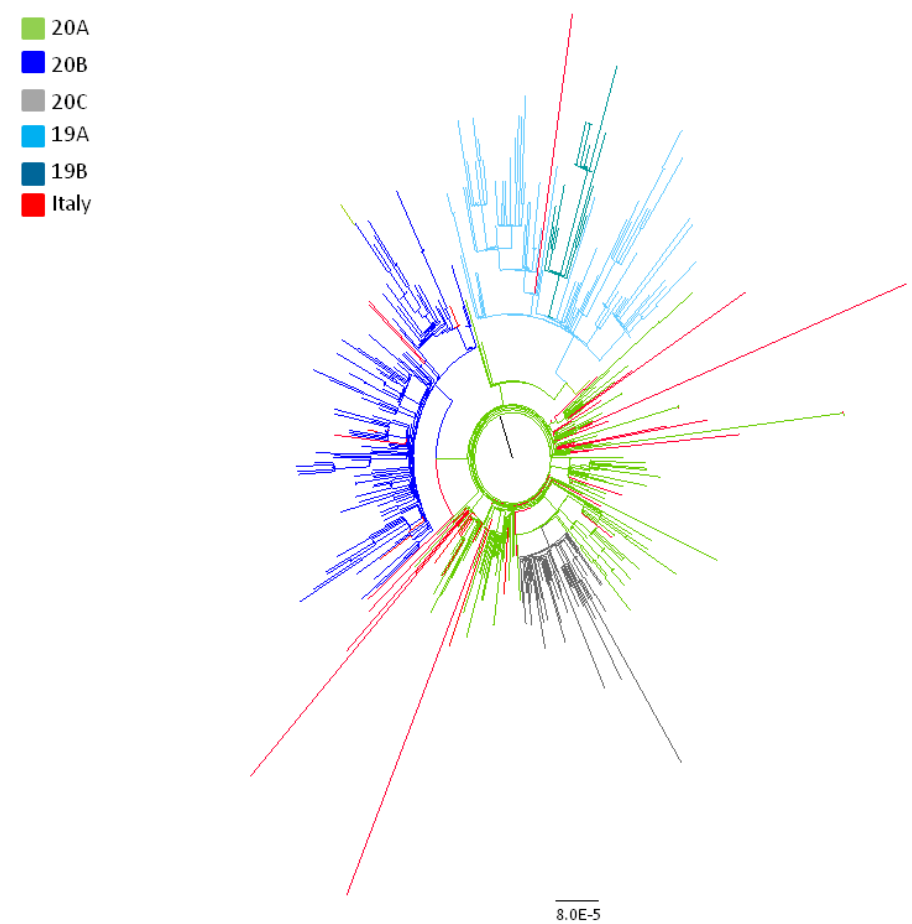

Figure 1. Maximum likelihood tree of the full dataset including 588 SARS-CoV-2 genomes. Nextstrain classification is indicated by colors as reported in the legend. Italian strains are highlighted in red.

Recently, new nomenclature systems have been proposed for the SARS-CoV-2 clades. The new lineage assignment of 62 Italian isolates is reported on Table 1 with the correspondence to other naming systems (old and new Nextstrain). All of our isolates belonged to the lineage B.1, only one isolate was classified as lineage B.

Table 1. Pangolin lineage classification of 62 Italian strains included in the study.

\begin{tabular}{cccccc}
\hline $\begin{array}{c}\text { Lineage } \\
\text { (Pangolin) }\end{array}$ & Total & \% & From & Nextstrain New & Nextstrain Old \\
\hline B & 1 & 1.6 & PD (1) & 19A & nd \\
\hline B.1 & 47 & 75.8 & $\begin{array}{c}\text { MI (15), PS(7), AN (1), MC (1) PD (8), BG (1), } \\
\text { CR (3), SI (3), AR (3), GR (1), BS (4) }\end{array}$ & 20A, nd & A2a \\
\hline B.1.1 & 11 & 17.7 & MI (4), PD (1), SI (4), GR (1), AR (1) & nd & A2a \\
\hline B.1.34 & 1 & 1.6 & MI (1) & A2a & A2a \\
\hline B.1.5 & 2 & 3.2 & MI (1), BG (1)
\end{tabular}

PD: Padua, MI: Milan, PS: Pesaro, AN: Ancona, MC: Macerata, BG: Bergamo, CR: Cremona, SI: Siena, AR: Arezzo, GR: Grosseto, BS: Brescia, nd: not determined. 


\subsection{Genetic Distances Analysis}

The overall mean $p$-distance between all the Italian isolates was 2.3 (SE:0.3) s/10,000 nts, corresponding to a mean of 6.4 (SE: 0.8) substitutions per genome. The non-synonymous distance $(\mathrm{dN})$ was 2.0 (SE: 0.4) non-syn s/10,000 non-syn nts while the overall synonymous mean distance (dS) was equal to 2.4 (SE: 0.05$)$ syn s/10000 syn $n t s(\mathrm{dN} / \mathrm{dS}=0.83)$. A higher heterogeneity was observed through months as, stratifying the genetic distances on the basis of the sampling time, we observed a higher heterogeneity among the strains isolated in February $(n=19)$ compared to those collected in March $(n=96)$ or April $(n=21)$ (Table 2).

Table 2. Mean genetic divergence within and between Italian strains according to the sampling time (substitutions per 10,000 sites).

\begin{tabular}{|c|c|c|c|c|c|c|c|c|c|}
\hline \multirow[t]{2}{*}{ Time } & \multicolumn{4}{|c|}{ Within } & \multirow[t]{2}{*}{ Time } & \multicolumn{4}{|c|}{ Between } \\
\hline & $\begin{array}{l}\text { p Distance } \\
\text { (SE) }\end{array}$ & $\begin{array}{l}\text { Nucleotide } \\
\text { (SE) }\end{array}$ & dS (SE) & $\mathrm{dN}(\mathrm{SE})$ & & $\begin{array}{l}\text { p Distance } \\
\text { (SE) }\end{array}$ & $\begin{array}{l}\text { Nucleotide } \\
\text { (SE) }\end{array}$ & dS (SE) & $\mathrm{dN}$ (SE) \\
\hline February & $\begin{array}{c}3.8 \\
(0.6)\end{array}$ & $\begin{array}{c}9.6 \\
(1.5)\end{array}$ & $\begin{array}{c}3.5 \\
(1.1)\end{array}$ & $\begin{array}{c}3.8 \\
(0.6)\end{array}$ & February vs. March & $\begin{array}{c}3.1 \\
(0.4)\end{array}$ & $\begin{array}{c}8.1 \\
(1.3)\end{array}$ & $\begin{array}{c}2.9 \\
(0.8)\end{array}$ & $\begin{array}{c}2.8 \\
(0.4)\end{array}$ \\
\hline March & $\begin{array}{l}1.9 \\
(0.3)\end{array}$ & $\begin{array}{c}5.4 \\
(0.8)\end{array}$ & $\begin{array}{c}2.2 \\
(0.5)\end{array}$ & $\begin{array}{c}1.5 \\
(0.4)\end{array}$ & March vs. April & $\begin{array}{c}2.3 \\
(0.3)\end{array}$ & $\begin{array}{c}6.6 \\
(0.8)\end{array}$ & $\begin{array}{c}2.1 \\
(0.6)\end{array}$ & $\begin{array}{c}2.0 \\
(0.5)\end{array}$ \\
\hline April & $\begin{array}{c}2.4 \\
(0.3)\end{array}$ & $\begin{array}{c}6.8 \\
(0.9)\end{array}$ & $\begin{array}{l}1.7 \\
(0.8)\end{array}$ & $\begin{array}{c}2.1 \\
(0.5)\end{array}$ & February vs. April & $\begin{array}{c}3.7 \\
(0.5)\end{array}$ & $\begin{array}{c}10 \\
(1.5)\end{array}$ & $\begin{array}{c}2.7 \\
(0.8)\end{array}$ & $\begin{array}{c}3.5 \\
(0.6)\end{array}$ \\
\hline
\end{tabular}

SE: Standard error, dS: synonymous distance, $\mathrm{dN}$ : non-synonymous distance.

\subsection{Differences in Amino Acids}

Considering only the non-synonymous mutations and comparing the Italian genomes with the common ancestor (China, EPI_ISL_402123), there were 159 amino acid substitutions affecting different viral genes, (112 in ORF 1a/1b, 19 in S, 12 in ORF 3a, 4 in M, 3 in ORF7a, 6 in N, and one each in Orf7b, 8 and 10) of which only $15(9.4 \%)$ were observed in 2 or more isolates, as summarized in Table 3. No amino acid changes were observed in the E gene. The previously described substitution D614G in the Spike protein was present in all the isolates belonging to the lineage B.1 and in the strain from Padua belonging to lineage $B$.

Table 3. SARS-CoV2 mutations identified in Italian strains.

\begin{tabular}{cccc}
\hline Genome Region & Mutation & $n$ /Total & Percentage (\%) \\
\hline & S443F & $2 / 135$ & 1.5 \\
H3076Y & $2 / 135$ & 1.5 \\
ORF 1ab & L3606F & $3 / 131$ & 2.3 \\
& P4715L & $133 / 136$ & 97.8 \\
& E5689D & $2 / 135$ & 1.5 \\
& R5919K & $2 / 123$ & 1.6 \\
\hline S & A570D & $2 / 129$ & 1.6 \\
& D614G & $128 / 130$ & 98.5 \\
ORF 3a & G1046V * & $3 / 134$ & 2.2 \\
\hline M & G251V & $3 / 134$ & 2.2 \\
\hline ORF 7a & D3G & $21 / 133$ & 15.8 \\
\hline \multirow{2}{*}{ N } & G70C & $2 / 134$ & 1.5 \\
\hline & R203K-G204R & $52 / 133$ & 39.1 \\
\hline
\end{tabular}

* mutation under significant selective pressure.

Considering the Italian isolates, only 1 site resulted under significant selecting pressure by three different methods (MEME, FEL, FUBAR): Site 1,046 in the Spike protein that was present in three isolates from Padua. This G1046V mutation is located in the S2 subunit, between heptad repeat 1 and 2 domains. Mutations R203K-G204R in N gene were always simultaneously detected. It appears that 
these mutations discontinue a serine-arginine (S-R) dipeptide by introducing a lysine in-between them, having impacts on structure and function in the mutated $\mathrm{N}$ protein.

Fifty-two sequences in our dataset carried these mutations, particularly 11 of the 59 whole genome newly-characterized; six of these were from Tuscany, four from Milan and one from Padua.

\subsection{Time Reconstruction of the SARS-CoV-2 Italian Lineage B.1 Phylogeny}

Root-to-tip regression analysis of the temporal signal from the Italian B.1 subset revealed a weak association between genetic distances and sampling days (a correlation coefficient of 0.31 and a coefficient of determination $\left(R^{2}\right)$ of $9.9 \times 10^{-2}$ ).

Comparison by BF test of the marginal likelihoods obtained by path sampling (PS) and stepping stone sampling (SS) of the strict vs. relaxed molecular clock (uncorrelated log-normal) showed that the second performed better than the former (strict vs. relaxed molecular clock $B F(P S)=-71.9$ and $\mathrm{BF}(\mathrm{SS})=-71.4$ for relaxed clock). Comparison of the different demographic models showed that the BSP and the exponential growth models best fitted the data (BSP vs. constant population size BF(PS) $=27.9$ and $\mathrm{BF}(\mathrm{SS})=30.2$ for $\mathrm{BSP}$; constant population size vs. exponential growth $\mathrm{BF}(\mathrm{PS})=7.3$ and $\mathrm{BF}(\mathrm{SS})=8.6)$ (Table S3).

The mean tMRCA of the tree root (Figure 2) was estimated at 107 days before present (BP) (95\%HPD: 91.2-113.1), corresponding to 11 January 2020 (from 5 January to 27 January). The tMRCA of the subclade including all the Italian sequences was estimated to be 92.4 (95\%HPD: 76.6-95) days BP, corresponding to 25 January (between 23 January and 10 February).

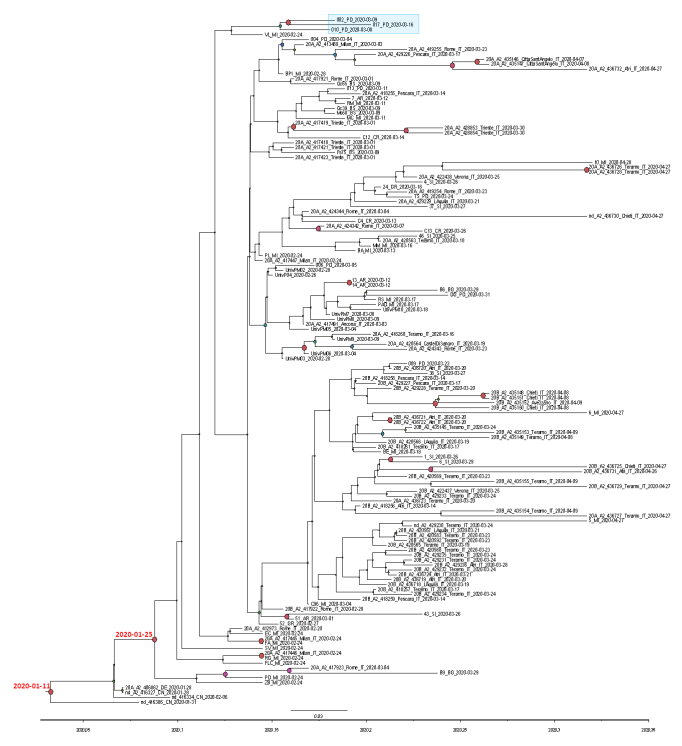

Figure 2. SARS-CoV-2 tree of 136 Italian strains plus one German and three Chinese isolates from Shanghai, showing statistically-significant support for clades along the branches (posterior probability $>0.7$ ). Large red and purple circles indicated highest posterior probability ranging from 1 to 0.9 . Calendar dates of the tree root and the Italian clade were showed in red. The light blue box highlighted the three Padua isolates carrying G1046V mutation in S protein.

The Bayesian tree of the Italian sequences showed 15 small significant subclades including two to ten isolates (Figure 2).

\subsection{Phylodynamic Analysis of the Italian Dataset}

The Bayesian skyline plot of the Italian isolates showed an increase in the number of infections in the period between late February and mid-March 2020, with a rapid exponential growth between 4 and 16 March when it reached a plateau continuing until the last sampling time (Figure 3). 


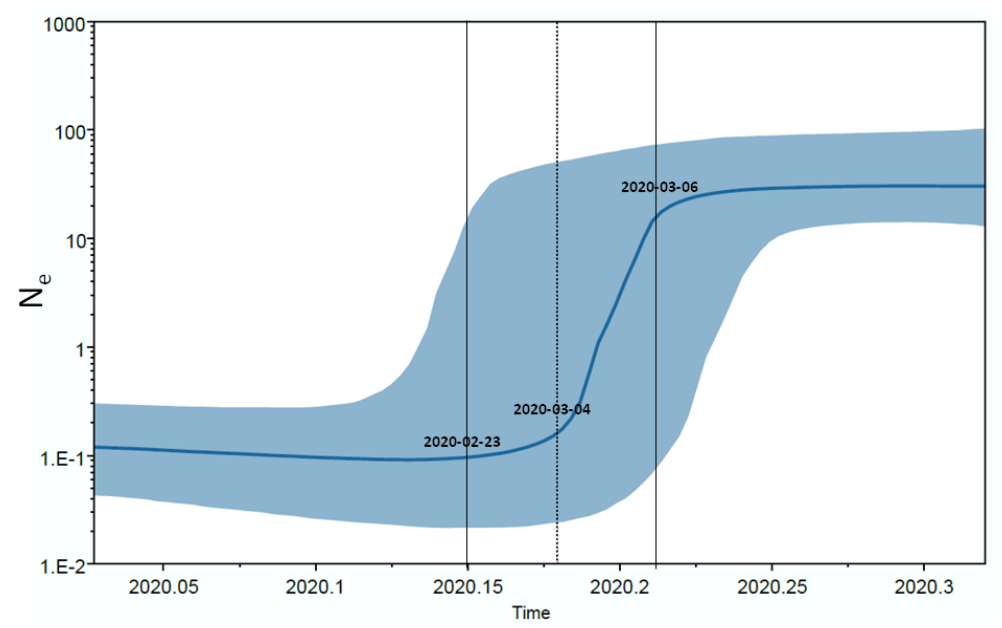

Figure 3. Bayesian Skyline plot of the SARS-CoV-2 outbreak. The $\mathrm{Y}$ axis indicates effective population size $(\mathrm{Ne})$ and the $\mathrm{X}$ axis shows the time in fraction of years. The thick solid line represents the median value of the estimates, and the grey area the 95\% HPD.

The Bayesian birth-death skyline plot of the $R_{e}$ estimates with $95 \% H P D$ with a single $R$ group (corresponding to $\mathrm{R}_{0}$ ) estimated a mean value of 2.25 (1.5-3.1). Figure 4 (panels a and $\mathrm{b}$ ) shows the changes of $R_{e}$ since the origin of the epidemic and suggests that $R_{e}$ was higher than 1 since the early days (mean initial $R_{e}=1.4,95 \% H P D: 0.08-2.9$ ). The curve started to grow in early February and peaked to a mean value of 2.3 (95\%HPD: 1.5-3.5) in the first half of March, and has since remained at this value. The curve obtained with three $R_{\mathrm{e}}$ groups showed a slight decrease at mid-March (Figure 4, panel b).
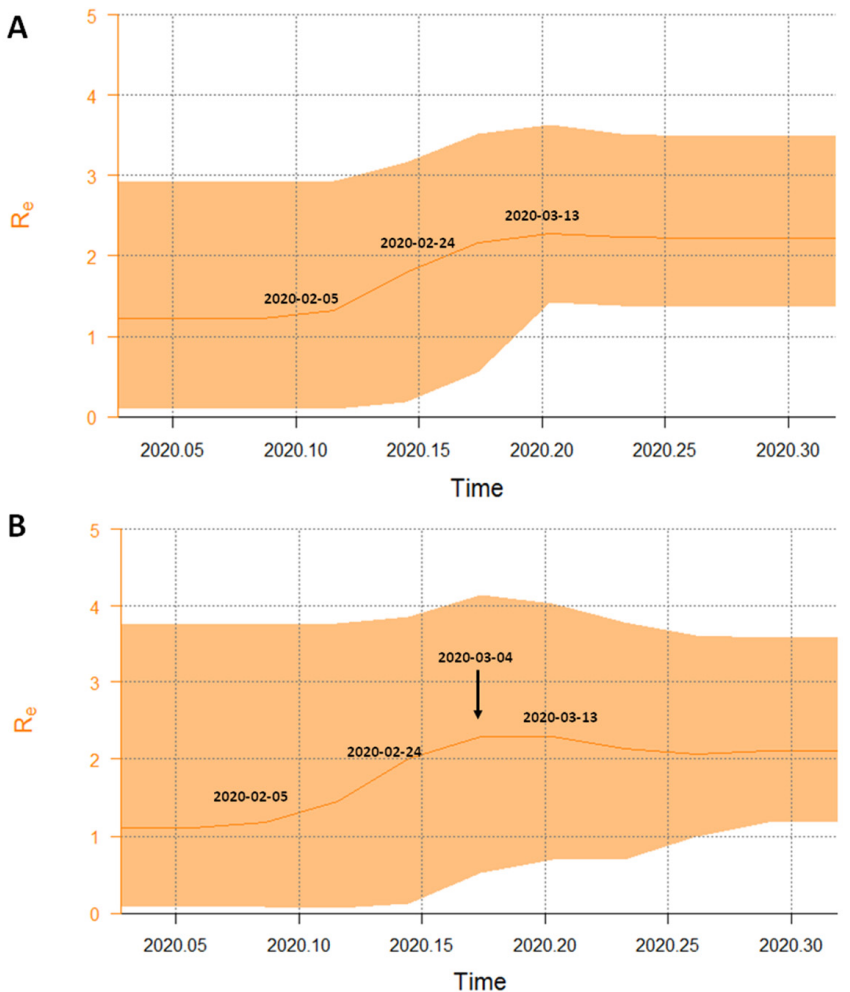

Figure 4. Part A: birth-death skyline plot of the SARS-CoV-2 outbreak allowing one $\mathrm{R}_{\mathrm{e}}$ intervals. Part B: birth-death skyline plot of the SARS-CoV-2 outbreak allowing three $\mathrm{R}_{\mathrm{e}}$ intervals. The curves and the orange areas show the mean $R_{e}$ values and their $95 \%$ confidence intervals. The $Y$ and $X$ axes indicate $R$ values and time in years, respectively. 
The origin of the epidemic was estimated at a mean 80.3 days BP (credibility interval: 60-109), corresponding to 7 February (between 9 January and 27 February). The recovery rate was estimated about 7.26 days (CI 4.7-16.0 days), and the transmission rate $(\lambda)$ increased from 71.7 to 115.96 in units per year (corresponding to a growth rate of 0.06 and 0.18 year $^{-1}$ ). On the basis of these data, the doubling time decreased from 5.1 days to 3.1 days in the period between early February and mid-March.

\section{Discussion}

Molecular tracing of SARS-CoV-2 coupled with advanced Bayesian and Maximum likelihood phylogenetic analysis provide detailed information about the epidemiology and evolution of emerging infections and helps to improve our understanding on the mechanisms of spreading of the epidemic.

In a previous study [1], we characterized the viral sequences obtained from the first three patients coming from the Codogno area who were hospitalized at the very beginning of the epidemic in Italy. The Codogno strains correlated with an isolate from an outbreak occurred in Bavaria around 20 January [4]. The present analysis shows that all but one of 62 SARS-CoV-2 sequences obtained from 22 February to the end of April in different Northern and Central Italian areas belong to a single clade, corresponding to the Pangolin lineage B.1, the old Nextstrain subclade A2a and the new Nextstrain clades 20A and 20B (https://nextstrain.org/blog/2020-06-02-SARSCoV2-clade-naming) [1,12]. About 1 out of 4 isolates were classified in different descendant lineages, always included in the main B.1 lineage (such as B.1.1 and B.1.5), most on a temporal basis, being these lineages more represented among the genomes sampled in the second half of March and April (9/14,64\%), while B.1 lineage was more represented in the genomes obtained in February and first half of March (33/47, 70.2\%).

This observation was also confirmed by other Italian studies [1,3]. The same clade is now the most widespread in the world and includes most of the published genomes [5]. The genetic distances among the Italian strains were relatively short, corresponding to an average of about 6.4 mutations per viral genome, even if single isolates may have a higher number of changes. After grouping the sequences according with the sampling months, while the within group mean genetic distances were higher in February compared to subsequent months, the genetic distance between different months increased with time. This observation confirms a continuous evolution of the viral genome (with the emergence of new divergent variants) mainly driven by genetic drift. No significant difference was observed between the non-synonymous and the synonymous substitutions $(\mathrm{dn} / \mathrm{ds}=0.8)$, suggesting the absence of relevant selective forces driving the evolution of the viral genome. This observation is further confirmed by the analysis of site-specific selective pressure in the Italian strains, which only showed a single site under significant positive selection in the $S$ protein (position 1046) observed in three strains from Padua. Including in the phylogenetic tree 3 isolates from Shanghai and one from the first patient of the Bavarian cluster, being at the root of the B.1 lineage, the dated tree obtained suggests that SARS-CoV-2 entered Italy between late January and early February 2020. This timing matches with the first autochthonous European cluster of SARS-CoV-2 transmission in Bavaria (Germany), originated on 20 January [1,4,21] by the introduction of a strain carried by the index patient coming from Shanghai, where the virus had been circulating since January. The skyline plot analysis of the Italian clade shows an exponential increase of the effective number of infections from late February to mid-March, in excellent agreement with the known epidemiological data (https://www.epicentro.iss.it/coronavirus/sars-cov-2-dashboard). In particular, a very rapid growth of the epidemic was detected between the beginning of March and the middle of the same month, when the curve reaches a plateau up to the end of sampling (27 April). The mean value of $R_{0}$ was estimated as 2.25 (1.5 to 3.1) in the entire period. A similar result was obtained by Stadler et al. on a smaller sample of 11 sequences mainly from patients with known travel history to Italy (https://virological.org/t/phylodynamic-analyses-based-on-11-genomes-fromthe-italian-outbreak/426). The estimated basic reproduction number $\left(R_{0}\right)$ for SARS-CoV-2 has ranged mainly from 2 to 4 , according to the different methods employed for the evaluation [22]. In Italy, values between 2.4 and 3.6 have been estimated in the early phase of COVID-19 epidemic before the control 
measures were taken [23-25]. Predictive mathematical models are fundamental to understand the dynamics of the epidemic, plan effective control strategies and verify the efficacy of those applied.

Using a birth-death skyline, we analyzed the changes of $R_{e}$ during the epidemic in Italy over the entire period. We observed that the $R_{e}$ was $>1$ since the first decade of February, suggesting that the infection was circulating within the population before the first notified (hospitalized) COVID-19 cases. The $R_{e}$ skyline plot reached a value of 2.3 in the first days of March, together with the rapid increase observed in the number of infections by BSP, and slightly decreased thereafter, in agreement with the official data on the course of the epidemic. Between February and March the estimated doubling time of the epidemic decreased from 5.1 to 3.1 days. This value was smaller than that obtained by us for the epidemic in China [26] and might be interpreted as a consequence of a delayed application of more stringent containment measures in Italy. In fact, a slight decrease of the $\mathrm{R}_{\mathrm{e}}$ value was observed only after mid-March, when a more rigorous social distancing was enforced across the entire country. The persistence of a $R_{e}$ value higher than one until April, in partial contrast with the epidemiological data (https://covstat.it/), could be due to the fact that our estimate was influenced by the circulation of the virus in the community, which is larger than the number of the officially registered clinical cases. It is well known that only a small minority of SARS-CoV-2 infections require hospitalization and that in Italy the number of cases of infection has widely exceeded the number of official reports. In a recent study, the prevalence of anti-SARS-CoV-2 antibodies in asymptomatic blood donors living in Milan was shown to increase from February to April, when the prevalence reached its maximum (about 7\%) (https://www.medrxiv.org/content/10.1101/2020.05.11.20098442v2). However, in Italy the numbers of active cases began to decrease only in the second half of April, when the present study had already been stopped. Further studies on extended data collection will be required to estimate the effects of the containment measures.

The only one genome characterized in our study not belonging to lineage B.1 was isolated in a 76-year-old man living in the province of Padua (Veneto), who survived to serious COVID-19 manifestations despite old age and the presence of several comorbidities. He denied any contact with infected subjects and did not travel abroad. This virus belongs to the same lineage (B) of the first 2 cases imported into Italy from the Hubei region, China, at the end of January 2020, before Italy suspended flights from China. The couple landed at the Milan airport and travelled to other locations in Northern and Central Italy before the onset of symptoms requiring hospitalization in Rome, but they had not travelled to Padua. Thus, the origin of such a strain remains unexplained and further investigations are underway to evaluate whether this strain may have played a role in causing an epidemic, at least locally. It would also be interesting to investigate whether the currently predominant strain was for some reasons more epidemic than the initial strain, or if the spread of the latter was limited by random factors.

In conclusion, our data show the importance of molecular and phylogenetic evolutionary reconstruction in the surveillance of emerging infections. Of note, it appears that the outbreak in Italy, which involved hundreds of thousands of people, is mainly attributable to a single introduction of the virus and its uncontrolled circulation for a period of about four weeks. These results reaffirm the strategic importance of continuous surveillance and timely tracing to define and rapidly implement effective containment measures for a possible second wave of the pandemic.

Supplementary Materials: The following are available online at http://www.mdpi.com/1999-4915/12/8/798/s1, Table S1: Data of Italian Patients characterized in the present study, Table S2: Accession IDs, sampling dates and location of sequences included in the dataset, Table S3: Comparison among different demographic models based on Path Sampling (PS) and Stepping Stone (SS) sampling.

Author Contributions: Conceptualization, A.L., G.Z., C.B., and M.G.; methodology, A.L., G.Z.; software, A.L., G.Z., and A.B.; formal analysis, A.L., G.Z., S.R., and A.B.; investigation, A.L., A.B., N.C., I.V., F.D., S.M., and F.C.; writing-original draft preparation, A.L., G.Z., and M.G.; writing-review and editing, A.L., G.Z., M.G., A.B., and C.B.; visualization, A.L., A.B., S.C., N.C., I.V., F.D., A.M.C., S.M., A.P., A.C. (Annapaola Callegaro), A.T., A.C. (Arnaldo Caruso), F.C., S.R., C.B., M.Z., E.V., M.C., M.G., and G.Z.; supervision, A.L., A.B., S.C., N.C., I.V., F.D., A.M.C., S.M., A.P., A.C. (Annapaola Callegaro), A.T., A.C. (Arnaldo Caruso), F.C., S.R., C.B., M.Z., E.V., M.C., M.G., 
and G.Z.; project administration, G.Z., C.B., and M.G.; funding acquisition, G.Z., M.G. All authors have read and agreed to the published version of the manuscript.

Funding: This research was funded by Fondo straordinario di Ateneo per lo Studio del Covid-19, University of Milan.

Acknowledgments: We acknowledge the authors and the originating and submitting laboratories of the GISAID sequences. The research was conducted under a cooperative agreement between Università degli Studi di Milano-Medicina del Lavoro e Clinica delle Malattie Infettive del Dipartimento di Scienze Biomediche e Cliniche "Luigi Sacco", Intesa Sanpaolo and Intesa Sanpaolo Innovation Center.

Conflicts of Interest: The authors declare no conflict of interest.

\section{Appendix A}

SCIRE collaborative Group: F Alessandrini ${ }^{1}$, M Andreoni ${ }^{2}$, G Antonelli ${ }^{3}$, S Babudieri ${ }^{4}$, P Bagnarelli ${ }^{5}$, S Bonora ${ }^{6}$, B Bruzzone ${ }^{7}$, G Brindicci ${ }^{8}$, P Carrer $^{9}$, F Ceccherini ${ }^{4}$, M Codeluppi $^{10}$, A Coluccello $^{11}$, N Coppola ${ }^{12}$, MG Cusi ${ }^{13,14}$, C Della Ventura ${ }^{9}$, L Di Sante ${ }^{5}$, S Di Giambenedetto ${ }^{15}$, G Di Perri ${ }^{6}$, V Fiore ${ }^{4}$, S Fiorentini ${ }^{16}$, D Francisci ${ }^{17}$, C Gandolfo $^{13}$, C Gervasoni $^{9}$, V Ghisetti ${ }^{6}$, R Greco $^{18}$, G Guarona $^{19}{ }^{\text {, M Iannetta }}{ }^{2}$, L Li Puma ${ }^{20}$, V. Malagnino ${ }^{2}$, G Mancuso $^{21}$, C Mastroianni $^{3}$, I Menozzi ${ }^{22}$, E Milano $^{8}$, A Miola $^{20}$, M Morganti $^{22}$, L Monno $^{8}$, G Noberasco $^{19}, \mathrm{G} \mathrm{Nunnari}^{21}$, V Onofri $^{1}$, A Orsi $^{19}$, MG Pierfranceschi ${ }^{11}$, S Pongolini ${ }^{22}$, D Ripamonti ${ }^{23}$, S Rubino ${ }^{4}$, L Ruggerone ${ }^{20}$, D Russignaga ${ }^{24}$, C Sagnelli ${ }^{12}$, M Sanguinetti ${ }^{15}$, L Sarmati $^{2}$, L Sasset ${ }^{25}$, E Scaltriti $^{22}$, R Schiavo $^{10}$, M Schioppa $^{26}$, S Testa ${ }^{11}$, C Turchi ${ }^{1}$, O Turriziani ${ }^{3}$, E Venanzi Rollo ${ }^{21}$, S Zanussi ${ }^{27}$, A Zoncada ${ }^{11}$

1. Section of Legal Medicine, Polytechnic University of Marche, Ancona, Italy

2. University of Rome Tor Vergata, Rome, Italy

3. Laboratory of Virology, Department of Public Health and Molecular Medicine, Sapienza University, Rome, Italy

4. Department of Medical, Surgical, and Experimental Sciences, University of Sassari, Viale San Pietro, Sassari, Italy

5. Department of Biomedical Sciences and Public Health, Virology and Legal Medicine Laboratories, Polytechnic University of Marche, Ancona, Italy

6. Clinic of Infectious Diseases, Amedeo di Savoia Hospital, University of Turin, Turin, Italy

7. Hygiene Unit, IRCCS AOU San Martino-IST, Genoa, Italy

8. University Hospital Consortium of the Polyclinic of Bari, Bari, Italy

9. Department of Biomedical and Clinical Sciences, ASST Fatebenefratelli Sacco, III Division of Infectious Disease, University of Milan, Milan, Italy

10. Diseases Unit of Infectious Diseases, Azienda Unità Sanitaria Locale, Piacenza, Italy

11. Unit of Infectious Diseases, Azienda Socio Sanitaria Territoriale Cremona, Cremona, Italy

12. Department of Mental Health and Public Medicine, Section of Infectious Diseases, University of Campania 'Luigi Vanvitelli', Naples, Italy

13. Department of Medical Biotechnologies, University of Siena, Siena, Italy

14. Microbiology and Virology Unit, Siena University Hospital, University of Siena, Siena, Italy

15. Institute of Clinical Infectious Diseases, Catholic University of Sacred Heart, Rome, Italy

16. Laboratory of Microbiology and Virology, Department of Molecular and Translational Medicine, Ospedali Civili, University of Brescia, Brescia, Italy

17. Infectious Diseases Clinic, Department of Medicine, University of Perugia, Perugia, Italy

18 Microbiology Unit, Clinical Pathology Complex Operative Unit, Sant'Anna e San Sebastiano Hospital, Caserta, Italy

19. Department of Health Sciences (DISSAL), University of Genoa, Genoa, Italy

20. Intesa San Paolo Innovation Center, Turin, Italy

21. Complex Operating Unit, Infectious Diseases, Policlinico Gaetano Martino, University of Messina, Messina, Italy

22. Risk Analysis and Genomic Epidemiology Unit, Experimental Prophylactic Zoo Institute of Lombardy and Emilia Romagna, Parma, Italy 
23. Infectious Diseases Unit, Azienda Socio Sanitaria Territoriale di Bergamo, , Papa Giovanni XXIII Hospital, Bergamo, Italy

24. Prevention and Protection Service, INTESA S.p.A., Turin, Italy

25. Infectious and Tropical Diseases, University Hospital of Padua,-Padua, Italy

26. Simple departmental operating unit of Genetics and molecular biology, AORN S. Anna e S. Sebastiano, Caserta, Italy

27. COS of Medical Oncology and Immunocorrelated Tumors, Oncological Reference Center, Aviano, Italy

\section{References}

1. Zehender, G.; Lai, A.; Bergna, A.; Meroni, L.; Riva, A.; Balotta, C.; Tarkowski, M.; Gabrieli, A.; Bernacchia, D.; Rusconi, S.; et al. Genomic characterization and phylogenetic analysis of SARS-COV-2 in Italy. J. Med. Virol. 2020, 29, 25794. [CrossRef]

2. Capobianchi, M.R.; Rueca, M.; Messina, F.; Giombini, E.; Carletti, F.; Colavita, F.; Castilletti, C.; Lalle, E.; Bordi, L.; Vairo, F.; et al. Molecular characterization of SARS-CoV-2 from the first case of COVID-19 in Italy. Clin. Microbiol. Infect. 2020, 26, 954-956. [CrossRef]

3. Stefanelli, P.; Faggioni, G.; Presti, A.L.; Fiore, S.; Marchi, A.; Benedetti, E.; Fabiani, C.; Anselmo, A.; Ciammaruconi, A.; Fortunato, A.; et al. Whole genome and phylogenetic analysis of two SARS-CoV-2 strains isolated in Italy in January and February 2020: Additional clues on multiple introductions and further circulation in Europe. Eurosurveillance 2020, 25, 2000305. [CrossRef]

4. Rothe, C.; Schunk, M.; Sothmann, P.; Bretzel, G.; Froeschl, G.; Wallrauch, C.; Zimmer, T.; Thiel, V.; Janke, C.; Guggemos, W.; et al. Transmission of 2019-nCoV Infection from an Asymptomatic Contact in Germany. N. Engl. J. Med. 2020, 382, 970-971. [CrossRef]

5. Korber, B.; Fischer, W.M.; Gnanakaran, S.; Yoon, H.; Theiler, J.; Abfalterer, W.; Hengartner, N.; Giorgi, E.E.; Bhattacharya, T.; Foley, B.; et al. Tracking Changes in SARS-CoV-2 Spike: Evidence that D614G Increases Infectivity of the COVID-19 Virus. Cell 2020. [CrossRef]

6. Kearse, M.; Moir, R.; Wilson, A.; Stones-Havas, S.; Cheung, M.; Sturrock, S.; Buxton, S.; Cooper, A.; Markowitz, S.; Duran, C.; et al. Geneious Basic: An integrated and extendable desktop software platform for the organization and analysis of sequence data. Bioinformatics 2012, 28, 1647-1649. [CrossRef]

7. Katoh, K.; Standley, D.M. MAFFT Multiple Sequence Alignment Software Version 7: Improvements in Performance and Usability. Mol. Biol. Evol. 2013, 30, 772-780. [CrossRef]

8. Kumar, S.; Stecher, G.; Li, M.; Knyaz, C.; Tamura, K. MEGA X: Molecular Evolutionary Genetics Analysis across Computing Platforms. Mol. Boil. Evol. 2018, 35, 1547-1549. [CrossRef]

9. Lott, M.; Murrell, B.; Golden, M.; Khoosal, A.; Muhire, B. RDP4: Detection and analysis of recombination patterns in virus genomes. Virus Evol. 2015, 1, 1. [CrossRef]

10. Posada, D. jModelTest: Phylogenetic Model Averaging. Mol. Boil. Evol. 2008, 25, 1253-1256. [CrossRef]

11. Stamatakis, A. RAxML version 8: A tool for phylogenetic analysis and post-analysis of large phylogenies. Bioinformatics 2014, 30, 1312-1313. [CrossRef]

12. Rambaut, A.; Holmes, E.; Hill, V.; O’Toole, Á.; McCrone, J.; Ruis, C.; Du Plessis, L.; Pybus, O.G. A dynamic nomenclature proposal for SARS-CoV-2 to assist genomic epidemiology. bioRxiv 2020. [CrossRef]

13. Suchard, M.A.; Lemey, P.; Baele, G.; Ayres, D.L.; Drummond, A.J.; Rambaut, A. Bayesian phylogenetic and phylodynamic data integration using BEAST 1.10. Virus Evol. 2018, 4, vey016. [CrossRef]

14. Bouckaert, R.R.; Vaughan, T.G.; Barido-Sottani, J.; Duchêne, S.; Fourment, M.; Gavryushkina, A.; Heled, J.; Jones, G.; Kühnert, D.; De Maio, N.; et al. BEAST 2.5: An advanced software platform for Bayesian evolutionary analysis. PLoS Comput. Boil. 2019, 15, e1006650. [CrossRef]

15. Rambaut, A.; Lam, T.T.; Carvalho, L.M.; Pybus, O.G. Exploring the temporal structure of heterochronous sequences using TempEst (formerly Path-O-Gen). Virus Evol. 2016, 2, vew007. [CrossRef]

16. Baele, G.; Lemey, P.; Bedford, T.; Rambaut, A.; Suchard, M.A.; Alekseyenko, A.V. Improving the Accuracy of Demographic and Molecular Clock Model Comparison While Accommodating Phylogenetic Uncertainty. Mol. Boil. Evol. 2012, 29, 2157-2167. [CrossRef]

17. Suchard, M.A.; Weiss, R.E.; Sinsheimer, J.S. Bayesian selection of continuous-time Markov chain evolutionary models. Mol. Boil. Evol. 2001, 18, 1001-1013. [CrossRef] 
18. Stadler, T.; Kühnert, D.; Bonhoeffer, S.; Drummond, A.J. Birth-death skyline plot reveals temporal changes of epidemic spread in HIV and hepatitis C virus (HCV). Proc. Natl. Acad. Sci. USA 2012, 110, 228-233. [CrossRef]

19. Li, Q.; Guan, X.; Wu, P.; Wang, X.; Zhou, L.; Tong, Y.; Ren, R.; Leung, K.S.; Lau, E.H.; Wong, J.Y.; et al. Early Transmission Dynamics in Wuhan, China, of Novel Coronavirus-Infected Pneumonia. N. Engl. J. Med. 2020, 382, 1199-1207. [CrossRef]

20. Walker, P.; Pybus, O.G.; Rambaut, A.; Holmes, E. Comparative population dynamics of HIV-1 subtypes B and C: Subtype-specific differences in patterns of epidemic growth. Infect. Genet. Evol. 2005, 5, 199-208. [CrossRef]

21. Spiteri, G.; Fielding, J.; Diercke, M.; Campese, C.; Enouf, V.; Gaymard, A.; Bella, A.; Sognamiglio, P.; Moros, M.J.S.; Riutort, A.N.; et al. First cases of coronavirus disease 2019 (COVID-19) in the WHO European Region, 24 January to 21 February 2020. Eurosurveillance 2020, 25, 2000178. [CrossRef]

22. Liu, Y.; A Gayle, A.; Wilder-Smith, A.; Rocklöv, J. The reproductive number of COVID-19 is higher compared to SARS coronavirus. J. Travel Med. 2020, 27, 27. [CrossRef]

23. D'Arienzo, M.; Coniglio, A. Assessment of the SARS-CoV-2 basic reproduction number, R0, based on the early phase of COVID-19 outbreak in Italy. Biosaf. Heal. 2020. [CrossRef]

24. Gatto, M.; Bertuzzo, E.; Mari, L.; Miccoli, S.; Carraro, L.; Casagrandi, R.; Rinaldo, A. Spread and dynamics of the COVID-19 epidemic in Italy: Effects of emergency containment measures. Proc. Natl. Acad. Sci. USA 2020, 117, 10484-10491. [CrossRef]

25. Yuan, J.; Li, M.; Lv, G.; Lu, Z.K. Monitoring transmissibility and mortality of COVID-19 in Europe. Int. J. Infect. Dis. 2020, 95, 311-315. [CrossRef]

26. Lai, A.; Bergna, A.; Acciarri, C.; Galli, M.; Zehender, G. Early phylogenetic estimate of the effective reproduction number of SARS-CoV-2. J. Med. Virol. 2020, 92, 675-679. [CrossRef] [PubMed]

(C) 2020 by the authors. Licensee MDPI, Basel, Switzerland. This article is an open access article distributed under the terms and conditions of the Creative Commons Attribution (CC BY) license (http://creativecommons.org/licenses/by/4.0/). 